\title{
Incidencia De La Destreza Listening A Través De Diálogos Y Closed Caption En El Aprendizaje Del Idioma Inglés
}

\section{Luis Oswaldo Guadalupe Bravo}

Magister en Lingüística y Didáctica de la Enseñanza de Idiomas Extranjeros

Docente de la Escuela Superior Politécnica de Chimborazo, Centro de Idiomas

Enrique Jesús Guambo Yerovi

Magister en Lingüística Aplicada al Aprendizaje del Idioma Inglés.

Docente de la Escuela Superior Politécnica de Chimborazo,

Centro de Idiomas

Luis Alberto Veloz Andrade

Magister en Administración para el Desarrollo Educativo

Docente de la Escuela Superior Politécnica de Chimborazo, Centro de Idiomas, Ecuador

\section{Salazar Calderón Edison Hernán}

Magister en Lingüística Aplicada al Aprendizaje del Ingles

Docente de la Escuela Superior Politécnica de Chimborazo,

Centro de Idiomas, Ecuador

\section{Mancero Orozco Washington Gustavo}

Magister en Administracion para el Desarrollo Educativo

Docente de la Escuela Superior Politécnica de Chimborazo, Centro de Idiomas, Ecuador

\section{Armas Pesantez Paul Rolando}

Magister en Lingüística Aplicada al Aprendizaje del Inglés

Docente de la Escuela Superior Politécnica de Chimborazo, Centro de Idiomas, Ecuador

\section{Doi: 10.19044/esj.2018.v14n2p204 URL:http://dx.doi.org/10.19044/esj.2018.v14n2p204}

\begin{abstract}
The objective of the present investigation was to make a diagnosis to the students of second year of baccalaureate parallel "H" of Pedro Vicente Maldonado High School in order to determine that the development of the skill of listening through activities like the dialogue and closed caption and the use of technologies of information and communication, contribute in the listening comprehension and therefore in the English language learning. The difficulty
\end{abstract}


in the listening comprehension was detected because of the student's low grades in the subject of the English. The research was scientific, quasiexperimental, and bibliographical, the information was collected through the technique of observation with a checklist that allowed the teacher to evaluate the student's performance. Both the activities and the parameters of the collation list were validated and verified by academic peers. The activities were organized in a timeline for their implementation; later the results were tabulated, analyzed, and interpreted, and subjected to the Chi square statistical test for the hypothesis test. Where it was shown that the application of activities with the help of the ICTs of the listening skill benefits the listening comprehension and thus the learning of the English language.

Keywords: Incidence, Listening, ICT, Learning, English

\section{Resumen}

El objetivo de la presente investigación fue realizar un diagnóstico a los estudiantes de segundo año de bachillerato paralelo " $\mathrm{H}$ " del Colegio Nacional Experimental Maldonado, para determinar que el desarrollo de la destreza del listening a través de actividades como el diálogo y closed caption con tecnologías de la información y comunicación, inciden en la comprensión auditiva y por ende en el aprendizaje del idioma inglés, pues se detectó la dificultad en la comprensión auditiva evidenciada en las bajas calificaciones en la asignatura del idioma inglés. La investigación fue de tipo científica, cuasiexperimiental y bibliográfica, donde la recolección de la información, fue a través de la técnica de la observación con una lista de cotejo, que le permitió al docente evaluar el desempeño del estudiante. Tanto las actividades como los parámetros de la lista cotejo fueron validados y verificados por pares académicos. Las actividades fueron organizadas en un cronograma para su aplicación; de ello los resultados obtenidos fueron tabulados, analizados e interpretados y sometidos a la prueba estadística Chi cuadrado para la comprobación de la hipótesis, donde se demostró que la aplicación de actividades con la ayuda de las Tecnologías de la Infomación y Comunicación TIC de la destreza de listening incide en la comprensión auditiva y por ende el aprendizaje del idioma inglés.

Palabras-claves: Incidencia, Listening, TIC, Aprendizaje, Inglés

\section{Introducción}

Si partimos del hecho que la comunicación es un proceso complejo, puesto que se presenta en forma espontánea e impredecible, es de suponer que ésta se torna aún más compleja cuando se emplea un idioma extranjero, en especial cuando se está aprendiendo la lengua extranjera. En el caso del 
aprendizaje del inglés como lengua extranjera, uno de los retos más frecuentes que afrontan los aprendices es el desarrollo de la habilidad de comprensión auditiva (Listening), hecho que se puede corroborar a través de la experiencia del docente del cuarto curso paralelo " $\mathrm{H}$ " del Colegio Experimental "Maldonado", mismo que al consultarle sobre la destrezas lingüísticas, manifestó que utiliza un lenguaje apropiado para crear buena comunicación con los alumnos en situaciones sociales y fuera del aula.

Realizando un análisis de los estudiantes del cuarto " $\mathrm{H}$ " curso del Colegio Maldonado de la Ciudad de Riobamba se detectó ciertas deficiencias en la pronunciación y comprensión auditiva, por lo que se consideró aplicar actividades de diálogo y closed caption mediante la destreza de listening en el aprendizaje del idioma inglés a través de las tecnologías de la comunicación e información que han sido aplicadas a la enseñanza-aprendizaje de las lenguas extranjeras satisfactoriamente, las cuales dinamizan la cobertura y efectividad del aprendizaje de un idioma. Se ha elegido los subtítulos (Closed Caption), como un sistema tecnológico asistido diseñado para proveer acceso a la información, la cual es transmitida a través de una señal codificada y descifrada por medio de una interfaz. Los programas y videos subtitulados crean un ambiente propicio de aprendizaje para que los estudiantes vean y escuchen las palabras de un determinado discurso al mismo tiempo y experimenten el significado de las dichas palabras gracias a la imagen que están viendo en la pantalla. De la misma manera se utilizó actividades como el diálogo a fin de ejercitar e incidir en el aprendizaje del idioma inglés.

Las habilidades receptivas, la comprensión auditiva y de lectura son fundamentales en el aprendizaje del inglés como lengua extranjera, dado que promueven la adquisición de estructuras gramaticales, el léxico, la pronunciación y demás aspectos indispensables en la consolidación de las bases idiomáticas, surge la necesidad de buscar e implementar nuevas estrategias para estimular el desarrollo de la comprensión auditiva.

Conociendo que el estado actual de la enseñanza de la destreza listening en la clase de inglés en secundaria no es muy satisfactoria, pues los resultados del rendimiento académico de los estudiantes de cuarto curso paralelo " $H$ " son insatisfactorios y el aporte de soluciones relacionadas al tema en cuestión. La intención de los autores es que los estudiantes de secundaria incrementen su nivel en el idioma inglés, a través de diversas actividades de escucha congruentes para estudiantes de quinto curso de secundaria.

\section{Metodología}

La investigación se enmarca en el área educativa bajo un diseño de investigación acción, y es de tipo descriptiva, las condiciones en que se desarrolló y explicó la relación entre variables. Además, es hipotética deductiva, porque describe las fases del método conjuntamente con sus etapas 
en la resolución de un problema práctico. En la investigación se indaga los aspectos teóricos y aplicados de medición, recolección, análisis y estadística de datos.

La población y muestra fueron de 45 estudiantes entre los 16 y los 17 años de edad de segundo año de bachillerato paralelo " $\mathrm{H}$ " del Colegio Experimental "Pedro Vicente Maldonado" de la provincia de Chimborazo. La distribución de la muestra corresponde a 15 estudiantes mujeres y 30 estudiantes hombres. Se trabajará con toda la población ya que el tamaño de la muestra es pequeña.

Se utilizó una técnica de observación con un test que fue verificado y validado por pares académicos del área. El test fue aplicado a los integrantes de la muestra que permitió conocer la percepción sobre la destreza del listening, actividades y ejercicios desarrollados en las clases de inglés. Aplicado los instrumentos, se establecieron los resultados, se codificaron, tabularon y elaboraron los cuadros de distribución, utilizando la técnica de porcentajes.

La representación de los datos fue mediante la estadística descriptiva utilizando frecuencias, gráficos, y porcentajes, mediante Microsoft Excel, de tal forma que se pueda visualizar claramente los resultados de cada una de las respuestas dadas por los participantes sobre datos generales, elementos, capacidades para el mejoramiento de la destreza listening en el aprendizaje del idioma inglés.

En primera fase se realiza una evaluación para conocer el estado inicial o nivel del conocimiento de Ingles, mediante un test que evalúa la destreza del listening dirigido a los estudiantes que representan la muestra de la investigación. En base a los resultados obtenidos en la primera fase se procedió al diseñó de actividades y ejercicios de comprensión auditiva (Listening), que involucren nuevas tecnologías de información y comunicación (TIC) con la inclusión y desarrollo de lecciones apoyadas en el uso del closed caption y diáñogos, con el fin de mejorar la comprensión auditiva. En la tercera fase, se puso en marcha las actividades y ejercicios de comprensión auditiva (Listening) diseñados para las clases de inglés. Finalmente se procedió evaluar los resultados obtenidos por los doscentes en las actividades y ejercicios implementados en las clases de inglés que involucran el uso de nuevas tecnologías como herramienta de apoyo para el desarrollo de la habilidad de Listening en los estudiantes. 


\section{Resultados}

Cuadro 1. Diagnóstico de listening y comprensión auditiva aplicando un diálogo en inglés.

\begin{tabular}{|c|c|c|c|}
\hline PARÁMETROS & $\begin{array}{l}\text { NOTAS } \\
\text { INDICADORES }\end{array}$ & $\begin{array}{l}\text { No. } \\
\text { ESTUDIANTES } \\
\text { FRECUENCIAS }\end{array}$ & PORCENTAJES \\
\hline $\begin{array}{l}\text { Demuestra total } \\
\text { comprensión de lo } \\
\text { escuchado. }\end{array}$ & $\begin{array}{l}\text { (Excelente) } \\
5\end{array}$ & 4 & $8,9 \%$ \\
\hline $\begin{array}{l}\text { Demuestra considerable } \\
\text { comprensión de lo } \\
\text { escuchado. }\end{array}$ & $\begin{array}{l}\text { (Muy buena) } \\
4\end{array}$ & 10 & $22,2 \%$ \\
\hline $\begin{array}{l}\text { Demuestra comprensión } \\
\text { parcial de lo escuchado. } \\
\text { (Buena) }\end{array}$ & $\begin{array}{l}\text { (Buena) } \\
3\end{array}$ & 9 & $20 \%$ \\
\hline $\begin{array}{l}\text { Demuestra poca } \\
\text { comprensión de lo } \\
\text { escuchado. (Buena) }\end{array}$ & $\begin{array}{l}\text { (Buena) } \\
2\end{array}$ & 6 & $13,3 \%$ \\
\hline $\begin{array}{l}\text { No comprende lo escuchado. } \\
\text { (Regular) }\end{array}$ & $\begin{array}{l}\text { (Regular) } \\
1\end{array}$ & 16 & $35,6 \%$ \\
\hline $\begin{array}{l}\text { No responde. No intentó } \\
\text { hacer la actividad. (Regular) }\end{array}$ & $\begin{array}{l}\text { (malo) } \\
0\end{array}$ & 0 & $0 \%$ \\
\hline TOTAL & & 45 & $100 \%$ \\
\hline
\end{tabular}

Fuente: Encuesta a estudiantes de segundo año de bachillerato paralelo "H" del Colegio Nacional Experimental "Maldonado" Realizado por: Guadalupe Luis, 2015

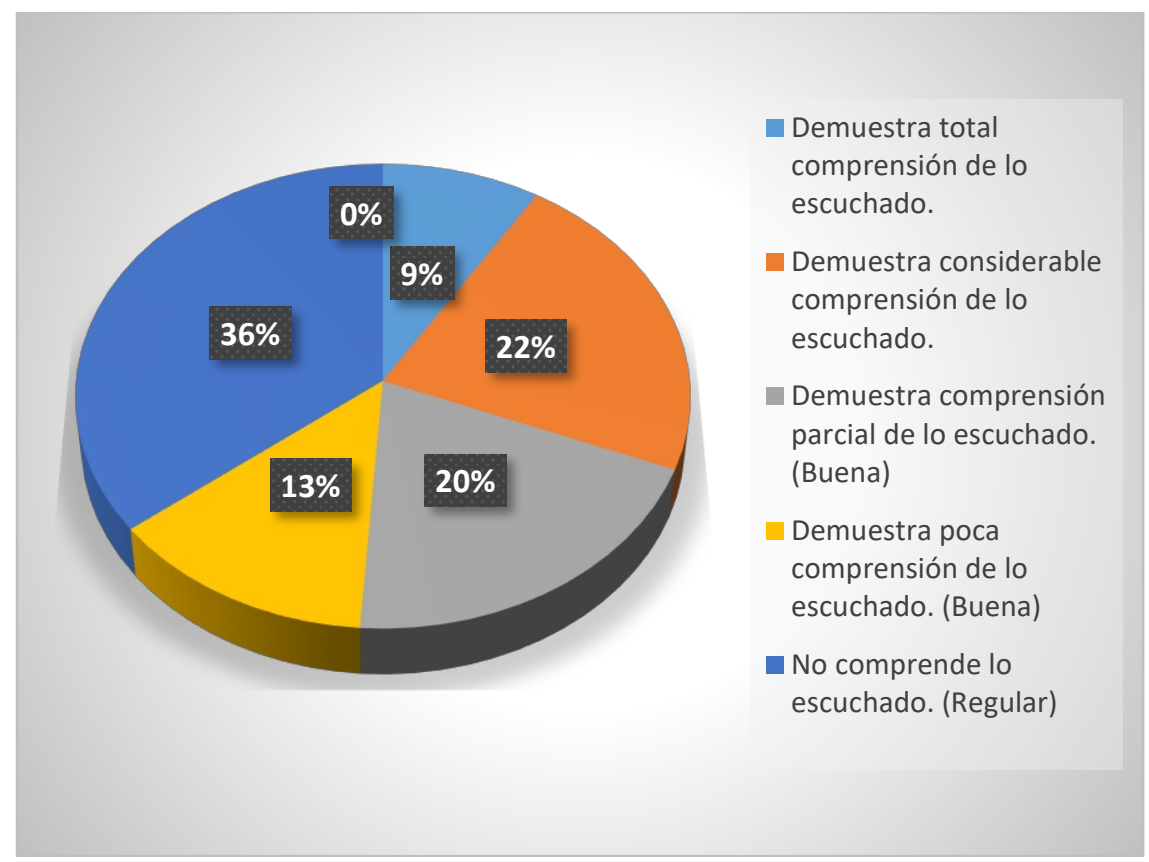

Gráfico 1. Diagnóstico de listening y comprensión auditiva aplicando un diálogo en inglés. 


\section{Análisis e Interpretación}

De la totalidad de estudiantes, el $36 \%$ demuestra que no comprende lo escuhado, el 9\%, demuestra una total comprensión de lo escuchado, el $22 \%$ demuestra considerable comprensión, un 20\% comprensión parcial; el 13,3\% poca comprensión; el 35,6\% no comprende lo escuchado; mientras que no existe frecuencias de la opción no responde.

En el diagnóstico realizado, la mayor frecuencia corresponde a los estudiantes que demuestran comprensión parcial de lo escuchado, considerando que es necesario buscar estrategias activas como el closed caption que permitirán un mejor desarrollo de la destreza del listening.

Cuadro 2. Diagnóstico de recursos usando en el listening

\begin{tabular}{|l|l|l|}
\hline PARÁMETROS & FRECUENCIAS & PORCENTAJES \\
\hline Música & 25 & $55,6 \%$ \\
\hline Videos & 12 & $26,7 \%$ \\
\hline Conversaciones & 5 & $11,1 \%$ \\
\hline No escuchan & 3 & $6,6 \%$ \\
\hline TOTAL & 45 & $100 \%$ \\
\hline
\end{tabular}

Fuente: Encuesta a estudiantes de segundo año de bachillerato paralelo " $\mathrm{H}$ " del Colegio Nacional Experimental "Maldonado"

Realizado por. Guadalupe Luis, 2015

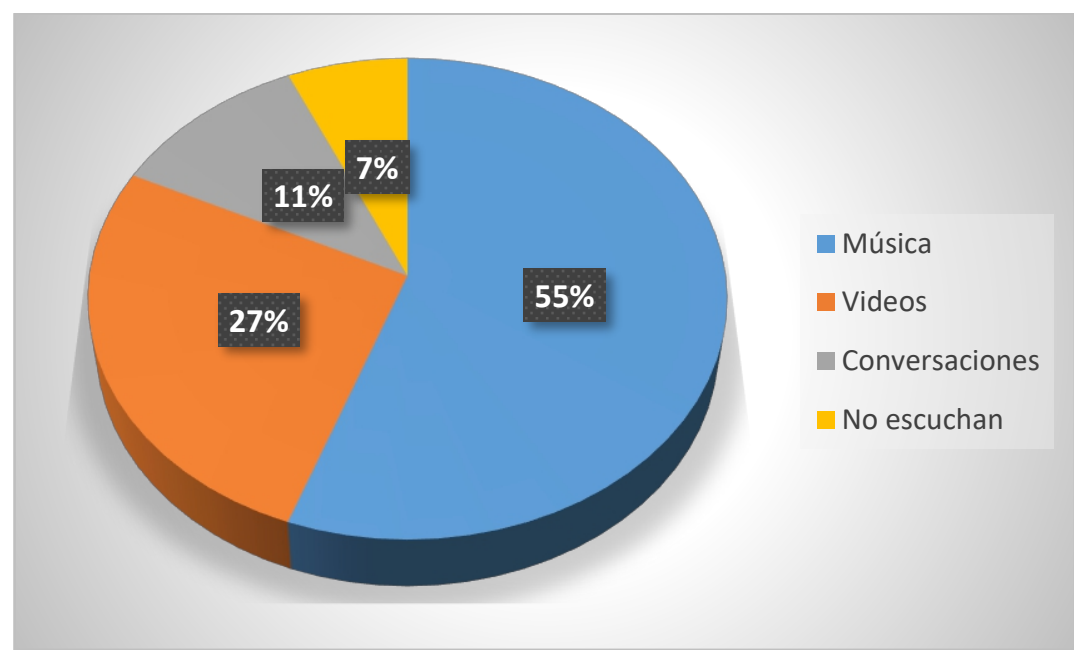

Gráfico 2. Diagnóstico para saber cuántos estudiantes realizan las siguientes actividades en inglés

\section{Análisis e Interpretación}

El 55\% de los estudiantes indican que escuchan música en inglés, el $27 \%$ expone que ven videos, el $11 \%$ escuchan conversaciones, mientras que el $7 \%$ no escuchan.

Más de la mitad de los estudiante realizan actividades en inglés, al escuchar música acción que no ayuda a desarrollar gradualmente la destreza del listening. 
Cuadro 3. Diagnóstico de ejercicio de completar una canción

\begin{tabular}{|l|l|l|}
\hline PARÁMETROS & FRECUENCIAS & PORCENTAJES \\
\hline Excelente & 4 & $8,9 \%$ \\
\hline Muy buena & 7 & $15,6 \%$ \\
\hline Buena & 19 & $42,2 \%$ \\
\hline Regular & 15 & $33,3 \%$ \\
\hline TOTAL & 45 & $100 \%$ \\
\hline
\end{tabular}

Fuente: Encuesta a estudiantes de segundo año de bachillerato paralelo "H" del Colegio Nacional Experimental "Maldonado"

Realizado por. Guadalupe Luis

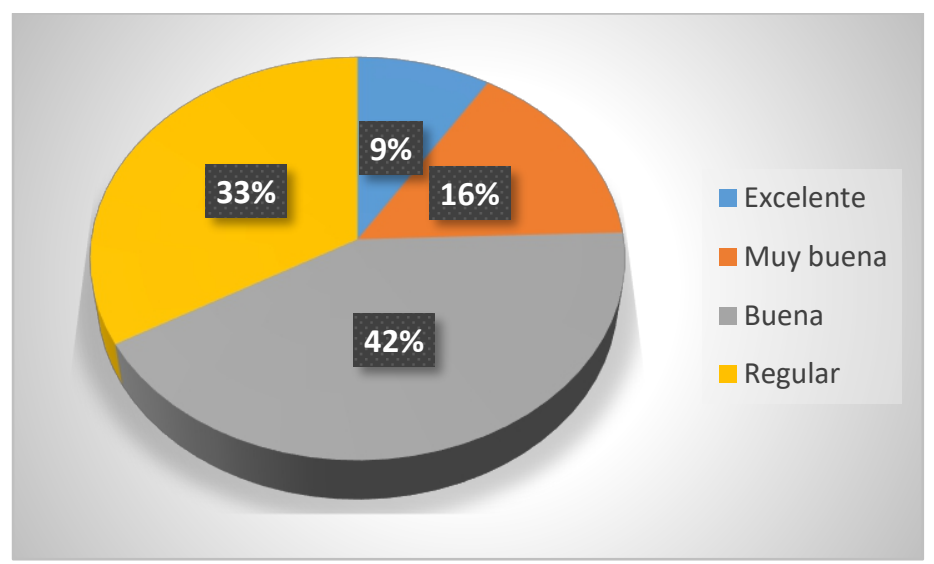

Gráfico 3. Diagnóstico de ejercicio de completar una canción

\section{Análisis e Interpretación}

Del total de los estuidnates, el 9\% tienen una valoración de excelente, el $16 \%$ muy buena, un $42 \%$ buena mientras que el $33 \%$ regular.

Como se puede evidenciar en los resultados los parámetros demuestran que la mayor parte de los estudiantes se involucran en las calificaciones de entre buena y regular, debiendo los docentes realizar este tipo de actividades para fortalecer el aprendizaje de esta área de estudio.

Cuadro 4. Diagnóstico closed caption para completar diez preguntas sobre un video

\begin{tabular}{|l|l|l|}
\hline PARÁMETROS & FRECUENCIAS & PORCENTAJES \\
\hline Excelente & 4 & $8,9 \%$ \\
\hline Muy buena & 8 & $17,8 \%$ \\
\hline Buena & 18 & $40 \%$ \\
\hline Regular & 15 & $33,3 \%$ \\
\hline TOTAL & 45 & $100 \%$ \\
\hline
\end{tabular}

Fuente: Encuesta a estudiantes de segundo año de bachillerato paralelo "H" del Colegio Nacional Experimental "Maldonado"

Realizado por. Guadalupe Luis 


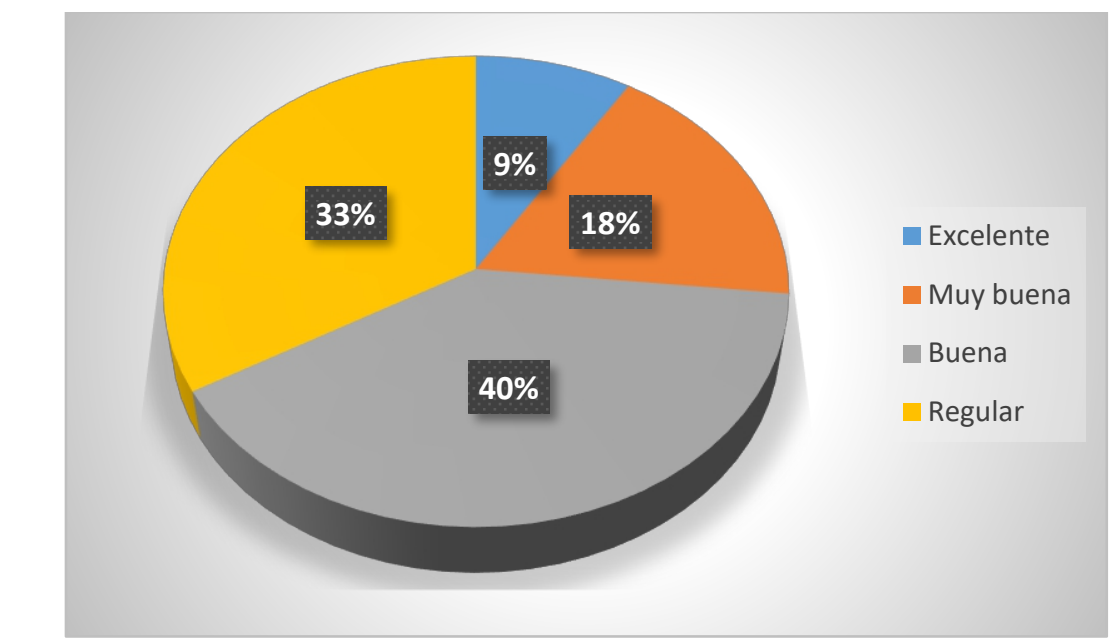

Gráfico 4. Diagnóstico closed caption para completar diez preguntas sobre un video

\section{Análisis e Interpretación}

Del total de los estudiantes encuestados, el 9\% tienen una valoración de excelente, el $18 \%$ muy buena, un $40 \%$ buena mientras que el $33,3 \%$ regular.

Es evidente que los estudiantes no pueden contestar un cuestionario con facilidad tomando en cuenta los resultados anotados de la encuesta donde expresan que la mayor parte de los estudiantes se encuentran en un valoración de buena y regular, considerando que se convierte en una debilidad para lograr el desarrollo de la destreza del listening en el aprendizaje del idioma inglés.

\section{Comprobación de la hipótesis}

Ho: La aplicación de la destreza de listening a través de actividades apoyadas en las TIC no incide en el aprendizaje del idioma inglés en los estudiantes de segundo año de bachillerato paralelo "H" Colegio Nacional Experimental Maldonado.

H1: La aplicación de la destreza de listening a través de actividades apoyadas en las TIC incide en el aprendizaje del idioma inglés en los estudiantes de segundo año de bachillerato paralelo " $\mathrm{H}$ " Colegio Nacional Experimental Maldonado.

El nivel de significación que se utilizó es $\alpha=0.05$; es decir la probabilidad de rechazar la Hipótesis nula Ho, cuando es verdadera. El nivel de confianza $\mathrm{B}=0.75$.

Con base a la información de la muestra, la formulación de la hipótesis y el cumplimiento de los supuestos; el estadístico de prueba que se utilizó es $\mathrm{CHI}$ cuadrado para determinar la aceptación o rechazo de la hipótesis Nula. 


$$
\chi_{C}^{2}=\sum \frac{\left(f_{o}-f_{e}\right)^{2}}{f_{e}}
$$

Donde:

$\chi^{C}=$ Es el valor de Chi cuadrado calculado.

fo $=$ Es la frecuencia observada

$\mathrm{fe}=$ Es la frecuencia esperada

\section{Análisis chi cuadrado de docentes y estudiantes en las encuestas matriz} de frecuencias observadas

\begin{tabular}{|c|c|c|c|c|}
\hline \multirow{3}{*}{\multicolumn{2}{|c|}{$\begin{array}{l}\text { V.INDEPENDIENTE } \\
\text { V. DEPENDIENTE }\end{array}$}} & \multirow{2}{*}{\multicolumn{3}{|c|}{$\begin{array}{l}\text { INCIDENCIA EN EL APRENDIZAJE DEL IDIOMA } \\
\text { INGLÉS }\end{array}$}} \\
\hline & & & & \\
\hline & & DIÁLOGO & CLOSED CAPTION & TOTAL \\
\hline & EXCELENTE & 4 & 4 & 8 \\
\hline 똠 & MUY BUENO & 10 & 8 & 18 \\
\hline$\stackrel{O}{\mathcal{N} Z}$ & BUENO & 9 & 18 & 27 \\
\hline 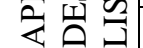 & REGULAR & 22 & 15 & 37 \\
\hline TOTAL & & 45 & 45 & 90 \\
\hline
\end{tabular}

Fuente: Encuesta a estudiantes de segundo año de bachillerato paralelo "H" del Colegio Nacional Experimental "Maldonado"

Realizado por: Guadalupe Luis, 2015

\section{Matriz de frecuencias esperadas}

\begin{tabular}{|c|c|c|c|c|}
\hline \multicolumn{5}{|c|}{ Cuadro 5. Frecuencias Esperadas } \\
\hline \multirow{2}{*}{\multicolumn{2}{|c|}{ V. DEPENDIENTE }} & \multicolumn{3}{|c|}{$\begin{array}{l}\text { INCIDENCIA EN EL APRENDIZAJE DEL IDIOMA } \\
\text { INGLÉS }\end{array}$} \\
\hline & & DIÁLOGO & CLOSED CAPTION & TOTAL \\
\hline \multirow{4}{*}{ 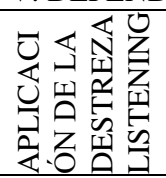 } & EXCELENTE & 4 & 4 & 8 \\
\hline & MUY BUENO & 9 & 9 & 18 \\
\hline & BUENO & 13,5 & 13,5 & 27 \\
\hline & REGULAR & 18,5 & 18,5 & 37 \\
\hline \multicolumn{2}{|l|}{$\frac{100=}{\text { TOTAL }}$} & 45 & 45 & 90 \\
\hline
\end{tabular}

Fuente: Encuesta a estudiantes de segundo año de bachillerato paralelo "H" del Colegio Nacional Experimental "Maldonado"

Realizado por: Guadalupe Luis, 2015 
Calculo del chi cuadrado

\begin{tabular}{|l|l|l|l|l|l|}
\hline CELDA & fo & fe & fo - fe & $\begin{array}{l}\text { (fo }- \text { fe }) \\
\text { Cuadro }\end{array}$ & $\frac{(f o-f e)^{2}}{f e}$ \\
\hline $\begin{array}{l}\text { Aplicación de la destreza listening } \\
\text { excelente, incide en el aprendizaje del } \\
\text { idioma inglés el diálogo. }\end{array}$ & 4 & 4 & 0 & 0 & 0 \\
\hline $\begin{array}{l}\text { Aplicación de la destreza listening muy } \\
\text { buena, incide en el aprendizaje del } \\
\text { idioma inglés el diálogo. }\end{array}$ & 10 & 9 & 1 & 1 & 0,111 \\
\hline $\begin{array}{l}\text { Aplicación de la destreza listening } \\
\text { buena, incide en el aprendizaje del } \\
\text { idioma inglés el diálogo. }\end{array}$ & 9 & 13,5 & -4.5 & $-20,25$ & 1,5 \\
\hline $\begin{array}{l}\text { Aplicación de la destreza listening } \\
\text { regular, incide en el aprendizaje del } \\
\text { idioma inglés el diálogo. }\end{array}$ & 22 & 18,5 & 3,5 & 12,25 & 0,662 \\
\hline $\begin{array}{l}\text { Aplicación de la destreza listening } \\
\text { excelente, incide en el aprendizaje del } \\
\text { idioma inglés el Closed Caption. }\end{array}$ & 4 & 4 & 0 & 0 & 0 \\
\hline $\begin{array}{l}\text { Aplicación de la destreza listening muy } \\
\text { buena, incide en el aprendizaje del } \\
\text { idioma inglés el Closed Caption. }\end{array}$ & 8 & 9 & 1 & 1 & 0,111 \\
\hline $\begin{array}{l}\text { Aplicación de la destreza listening } \\
\text { buena, incide en el aprendizaje del } \\
\text { idioma inglés el Closed Caption. }\end{array}$ & 18 & 13,5 & 4.5 & 20,25 & 1,5 \\
\hline $\begin{array}{l}\text { Aplicación de la destreza listening } \\
\text { regular, incide en el aprendizaje del } \\
\text { idioma inglés el Closed Caption. }\end{array}$ & 15 & 18,5 & $-3,5$ & $-12,25$ & 0,662 \\
\hline & 45 & 45 & & & \\
\hline
\end{tabular}

Calculo del valor para el $\chi^{2}$

$\chi_{C}^{2}=\sum \frac{\left(f_{o}-f_{e}\right)^{2}}{f_{e}}$

$\chi^{2}=4,546$

El valor de $\chi^{T}$ se lo determinó con los siguientes grados de libertad.

GL: (C-1) (F-1)

GL: (2-1) (4-1)

GL: 3

$\chi_{T}^{2}: 1,923$ 
Con el $75 \%$ de significancia estadística entramos a la tabla y obtenemos un chi cuadrado de la tabla $\chi_{T}^{2} 1,923$

Con $v$ = grados de libertad y con un nivel de significación de ${ }^{\propto: 0.90}$ (nivel de confianza del $75 \%$ ) se tiene que el valor teórico de Chi cuadrado es:

$$
\chi_{\alpha, v}^{2}=\chi_{0,75}^{2}=4,546
$$

4,546 es > que 1,923, por lo tanto, cae en la zona de rechazo, entonces rechaza la hipótesis nula que con la aplicación de la destreza de listening no existe incidencia en el aprendizaje del idioma inglés de los estudiantes de segundo año de bachillerato paralelo " $H$ " Colegio Nacional Experimental Maldonado.

\section{Conclusión de tipo estadístico de docentes y estudiantes}

\section{Chi-cuadrado}

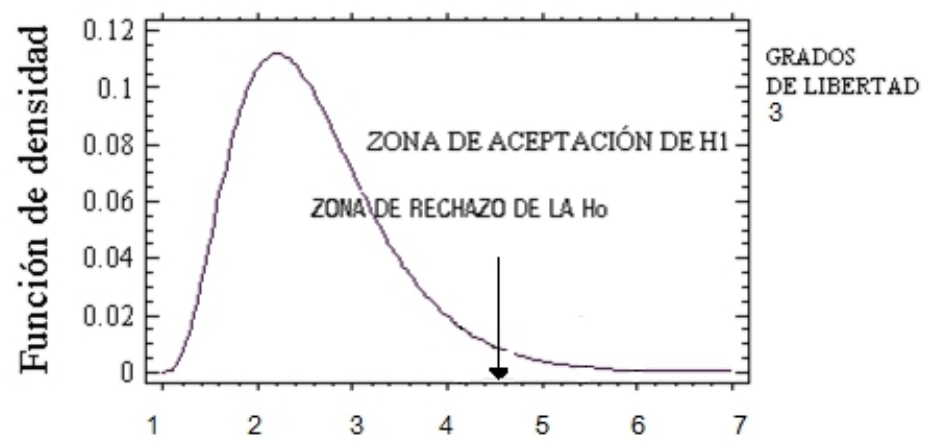

Eje $x$

Debido a que $\mathrm{X}^{\mathrm{C}}=4,526>\mathrm{X}^{2}=1,923$ este se encuentra en la zona de rechazo $\mathrm{H}^{0}$ y por lo tanto se acepta la hipótesis $\mathrm{H}^{1}$ que es la hipótesis de investigación.

Con el nivel de confianza del $75 \%$ podemos decir la destreza de listening a través de actividades como el diálogo y closed caption, incidieron en el aprendizaje del idioma inglés en los estudiantes de segundo año de bachillerato paralelo "H" Colegio Nacional Experimental Maldonado.

Con los resultados obtenido en cada una de las actividades con el apoyo de las TIC aplicadas a los estudiantes se demuestra que el desarrollo de la destreza listening incide en la comprensión auditiva del aprendizaje del idioma inglés de los estudiantes de segundo año de bachillerato paralelo " $\mathrm{H}$ " 
del Colegio Nacional Experimental "Pedro Vicente Maldonado" ubicado en la ciudad de Riobamba, provincia de Chimborazo. Durante el periodo 2010 - 2011, con todos estos antecedentes consideramos que la hipótesis de investigación es comprobada.

\section{Discusión}

La destreza es la expresión del saber hacer en los estudiantes y caracteriza el "dominio de la acción"; y en el concepto curricular realizado se le ha añadido criterios de desempeño, los que orientan y precisan el nivel de complejidad sobre la acción: pueden ser condicionantes de rigor científico cultural, espaciales, temporales, de motricidad y otros(Bonafé, 2002).

Las destrezas constituyen el referente principal para que el profesorado elabore la planificación para el proceso de enseñanza aprendizaje. Sobre la base de su desarrollo y su sistematización, se graduarán de forma progresiva y secuenciada los conocimientos conceptuales e ideas teóricas, con diversos niveles de integración y complejidad. (“APRENDYDESARRPROFESIONAL.pdf”, s. f.)

Para comprender lo que se escucha, así como para comprender lo que se lee, es necesario prestar atención. A veces resulta difícil mantenerse atento. $\mathrm{Si}$ se identifican las causas que provocan las distracciones, es posible que se pueda hacer algo para evitarlas o controlarlas. Los factores que causan distracción pueden ser personales o situacionales.

Quien sabe escuchar estas señales, incrementa su poder de persuasión que se mide por el grado de influencia. Los que se "meten en el bolsillo a la gente" arman agendas, median en conflictos, porque saben conectarse. La clave es aprender a realizar un identikit auditivo.(Redacción, 2016)

El aprendizaje de una lengua extranjera abarca el desarrollo de cuatro destrezas importantes que corresponden a escribir, hablar, leer y escuchar; de las cuales, las dos primeras han sido consideradas por mucho tiempo por parte de los académicos como destrezas activas y las dos últimas como destrezas pasivas.

Sin embargo, este concepto ha cambiado principalmente a nivel de la comprensión auditiva, puesto que ahora es considerada una habilidad lingüística que los estudiantes pueden desarrollar de manera activa evitando caer en el error de oír en vez de escuchar; de tal forma, que según Peris consiste en "desarrollar una actividad de interpretación, que tiene sus propias técnicas y estrategias." Otro autor que confirma el nuevo concepto dinámico de la comprensión auditiva es Gary Buck, autor de Assessing Listening, quien define "el Listening como un proceso complejo cognitivo, individual e inferencial." Desde el punto de vista de James, la comprensión auditiva "no es solo una destreza, sino una serie de destrezas marcadas por el hecho de involucrar la percepción auditiva de signos orales", lo cual se ve respaldado 
por el concepto de Canale y Awain, quienes entienden que la destreza de comprensión auditiva "se compone de diferentes subcompetencias: una competencia gramatical, una competencia sociolingüística y sociocultural, una competencia discursiva y finalmente, una competencia estratégica (Canale, Awain, 1996)

Por ser un proceso dinámico, encontrar una única definición para la comprensión auditiva es una tarea difícil, a tal punto que existen diversas concepciones diferentes todas entre sí. Por ejemplo, Wipf, en el artículo Strategies for Teaching Second Language Listening Comprensión, definió la comprensión auditiva como "un proceso mental invisible difícil de describir en el que las personas que escuchan deben discriminar entre los diferentes sonidos, comprender el vocabulario y las estructuras gramaticales, interpretar el énfasis y la intención, y retener e interpretar todo esto tanto dentro del contexto inmediato como de un contexto socio-cultural más amplio." De acuerdo con este autor, la definición de la comprensión auditiva presenta un enfoque estructural del idioma, es decir, se evidencia la importancia de los componentes fonológicos y léxicos de la lengua extranjera (WIPF, 1984).

Por otro lado, existen definiciones encaminadas hacia un punto de vista social, en el que la importancia radica en la interacción entre el emisor y el receptor, como es el caso de la definición de Rost, para quien la comprensión auditiva es "un proceso de recibir lo que el emisor en realidad expresa (la orientación receptiva); construir y representar el significado (la orientación constructiva); negociar el significado con el emisor y responder (la orientación colaborativa); y crear significado a través de la participación, la imaginación y la empatía (la orientación transformativa) (Rost, 2002).

Queda claro entonces, que la destreza de comprensión auditiva como un proceso activo involucra la comprensión de: los fonemas, el énfasis, la entonación, la velocidad y el significado de aquello que se está escuchando. Y para que este proceso ocurra, según Douglas Brown en su texto Assessing Listening describe que cuando escuchamos "en el cerebro tienen lugar cuatro procesos: reconocemos sonidos; simultáneamente determinamos el tipo de discurso del que se trata; interpretamos el mensaje literal e intentamos inferir a partir de lo explícito y finalmente, seleccionamos información: sólo retenemos la información que consideramos más importante o relevante (en donde interviene la memoria a largo plazo)."

De acuerdo con Galvin, el proceso de comprensión auditiva también requiere de cuatro pasos fundamentales: "el primer paso es la recepción (receiving) y consiste en poner nuestro sentido del oído a trabajar. En este primer, paso la persona "decide" escuchar, o lo que es lo mismo, la persona empieza a clasificar entre lo que constituye únicamente "ruido" y lo que él o ella realmente desea o debe escuchar. El segundo paso es la interpretación. En este nivel, el receptor activa todas sus experiencias y vivencias para 
decodificar lo que acaba de escuchar. El paso número tres es la evaluación, el cual se basa en decidir qué hacer con el mensaje. Y, por último, el paso final en este proceso es la respuesta, que no es necesariamente una respuesta oral, ya que puede ser perfectamente cualquier otra reacción tal como sonreír, asentir, realizar alguna otra acción o escribir algo." (Galvin, 1988).

Para Adell las nuevas tecnologías son "el conjunto de procesos y productos derivados de las nuevas herramientas (hardware y software) soportes de la información y canales de comunicación relacionadas con el almacenamiento, procesamiento y transmisión digitalizados de datos" (Adell, 2000). Esta definición se complementa con el apartado de González, Gisbert y otros, que insisten en que estas herramientas "tienen un carácter innovador y que están dando lugar a nuevos procesos culturales y educativos" (González, 2000).

Por su parte, Perez Marqués considera que para el apropiado uso de las tecnologías de la información se debe contar "con un conocimiento básico del sistema informático (software \& hardware)". Así mismo, considera que se debe contar con unas competencias básicas que permitan que los estudiantes se beneficien de las TIC (Marqués, 2000).

El Closed Caption muestra el diálogo, la narración y los efectos de sonido de un programa o video como palabras en una pantalla, muy parecido a los subtítulos de una película. Sin embargo, a diferencia de las películas subtituladas, el Closed Caption le permite al estudiante escoger o no si los subtítulos son mostrados en la pantalla. Cientos de miles de personas también se benefician del Closed Caption, entre las que encontramos a personas que están aprendiendo una segunda lengua, niños y jóvenes que están aprendiendo a leer, estudiantes con problemas de aprendizaje, personas analfabetas e incluso personas que ven televisión en ambientes ruidosos como restaurantes, bares y aeropuertos.

\section{Conclusion}

Con la evaluación del estado inicial o nivel del conocimiento de inglés de los estudiantes se conoció la debilidad en la comprensión auditiva evidenciada en las bajas calificaciones en la asignatura del idioma inglés.

Con el diseño de actividades y ejercicios de comprensión auditiva (Listening), a través de diálogos y closed caption apoyadas con las tecnologías de información y comunicación (TIC) se obtuvo instrumentos que permitieron evaluar la incidencia en el aprendizaje del idioma inglés.

Con los resultados obtenido en las actividades del diálogo y closed caption y con el apoyo de las TIC aplicadas a los estudiantes se demuestra que el desarrollo de la destreza Listening incide en la comprensión auditiva del aprendizaje del idioma inglés de los estudiantes de segundo año de bachillerato paralelo "H" del Colegio Nacional Experimental "Pedro Vicente 
Maldonado" ubicado en la ciudad de Riobamba, provincia de Chimborazo, con todos estos antecedentes consideramos que la hipótesis de investigación es comprobada.

\section{Recomendaciones}

Es indispensable que los docentes de inglés realicen diálogos como una de las actividades pedagógicas que contribuyan al desarrollo de destrezas de escuchar para lograr el aprendizaje del inglés.

Los docentes deben poner mayor énfasis en la aplicación de diálogos, hojas de trabajo tales como completar y enlazar, canciones, closed caption referentes a la asignatura de inglés buscando alternativas de solución para el problema investigado.

\section{References:}

1. ACOSTA MATOS, Héctor Alejandro. The effects of Closed Caption upon the Listening comprehension of students learning English as a second language in an intermediate school in Puerto Rico. Universidad de Puerto Rico. 2003.

2. APRENDYDESARRPROFESIONAL.pdf. (s. f.). Recuperado a partir de

http://www.oei.es/historico/metas2021/APRENDYDESARRPROFE SIONAL.pdf

3. BASTIDAS, Jesús A. Opciones Metodológicas para la Enseñanza de Idiomas. Editorial Saba. 1993. p. 30; 35; 165.

4. BONAFÉ, J. M. (2002). Políticas del libro de texto escolar. Ediciones Morata.

5. BORRÁS, I. \& LAFAYETTE. Effects of multimedia courseware subtitling on the speaking performance of college students of French. En: The Modern Language. Journal 78,1: 61-75

6. BROWN H. Douglas. Principles of Language Learning and Teaching, 4th edition. New York: Longman. 2000. p. 30-49

7. BROWN, Douglas. Language Assessment. Principles and Classroom Practices. New York: Pearson Longman. 2004. p. 54

8. BUCK, Gary. Assessing Listening. Cambridge University Press. 2001 Cabrero Almenara, Julio. Nuevas Tecnologías Aplicadas a la Educación. Madrid: Mc. Graw Hill. 2007

9. CABRERO ALMENARA, Julio. Nuevas Tecnologías Aplicadas a la Educación. Madrid: Mc. Graw Hill. 2007

10. CAJIDE, J. Diseño y Técnicas de Evaluación de Estrategias de Enseñanza-Aprendizaje. En: Revista Española en Pedagogía. Bordon. Vol. 50. (1992) p. 101-112 CANALE, M \& AWAIN, M. Fundamentos 
Teóricos de los Enfoques Comunicativos. Teoría y Práctica de la Educación. En: Revista Signos. Chile. Vol 3. No.17 (1996); p.54-69

11. DOMINGUEZ, Rocío. La Investigación Acción como Método de Investigación para Docentes. 2003. Recuperado el 10 de Marzo de 2009.

12. ELLIOT, J. El cambio educativo desde la investigación-acción. Madrid: Morata. 1993. p. 69

13. ELLIS, R. Understanding Second Language Acquisition. Oxford: Oxford University Press. 1992. p. 34.

14. EVANS, T. Understanding learners in open and distance education. Londres: Kogan Page, 1993

15. FINOCCHIARIO, M. \& BRUMFIT. The Functional - Notional Approach: From theory to practice. New York: Oxford University Press. 1983

16. GALVIN, K. Listening by Doing: Developing Effective Listening Skills. Lincolnwood, 1ll.: National Textbook Company. 1988

17. GARDNER, R.C. y MACLNTYRE, P. D. "A student's contributions to second language leaming. Part 11: Affective variables." Language Teaching, 26. 1993. p. 1-11.

18. HALLIDAY, M.A.K. Language Structure and Language Function: New Horizons in Linguistics. Londres: Penguin books. 1970

19. HART, E y BOND, M. Action Research for Health and Social Care: A Guide to Practice. Open University Press, Buckingham. 1995. p. 371 378.

20. HERNÁNDEZ, R. FERNÁNDEZ, C. y BAPTISTA, P. Metodología de la investigación. México: Editorial Mc Graw Hill. 1998. p. 243.

21. HYMES, D. On Communicative Competence. Sociolinguistics. Londres: Penguin Books. 1972

22. JAMES, C.J. Are you Listening?: The Practical Components of Listening Comprehension. En: Foreign Language Annals, 17. 1984. p. 339-342.

23. JENSEN, E.D. Video didactics and the acquisition of a foreign language. En: D. Alberechtsen, B. Henriksen, I. Mess y E. Poulsen (Eds.). Perspectives on foreign and second language pedagogy. Odense University Press. 1998. p. 253-262

24. LYNCH, T \& MENDELSOHN,D. Listening. En: SCHMITT, Norbert. An Introduction to Applied Linguistics. New York: Oxford. 2002.p.193-210

25. McLAUGHLIN, Barry. Theories of Second Language Learning. London: Edward Arnold. 1991.

26. MET \& GALLOWAY. Research in Foreign Language Currículo. New Cork: Macmillan.1996 
27. NUNAN, D. Listening in Language Learning. En: The Language Teacher Online. Available October, 2004: [http://jaltpublication.org/ttt/files/97/sep/nunan.html.]

28. Redaccion. (2016, agosto 17). Emprendedores.News. Recuperado 6 de octubre de 2017, a partir de http://emprendedoresnews.com/tips/aprender-a-escuchar.html

29. STERN, H.H. Fundamental Concepts of Language Teaching 9th Impression. Oxford: Oxford University Press. 1996. p. 14.

30. TORREALBA, Francisco \& MARTÍNEZ, María Kristina. Las parábolas como estrategias de enseñanza para la producción de textos escritos, dirigido a alumnos del quinto grado. Venezuela: 2007.p.50

31. WILKINS, D.A. Linguistics in Language Teaching. London: Edward Arnold Ltd. 1972

32. WILLIAMS, Helen y THORNE, David. The value of teletext subtitling as a medium for language learning. 2000. Pág. 4-5

33. WIPF, J. Strategies for Teaching Second Language Listening Comprehension. En: Foreign Language Annals, 17. 1984. p. 345- 348.

34. YORK, Heather. SPECIAL REPORT: Understanding video captioning rules. Overland Park. Octubre 2005. Vol. 47, Iss. 10; p. 85 